\title{
The Drosophila Jun-N-terminal kinase is required for cell morphogenesis but not for DJun-dependent cell fate specification in the eye
}

\author{
Juan R. Riesgo-Escovar, Mario Jenni, Andreas Fritz, ${ }^{1}$ and Ernst Hafen ${ }^{2}$ \\ Zoologisches Institut, Universität Zürich, Winterthurerstrasse 190, CH-8057 Zürich, Switzerland
}

\begin{abstract}
We cloned and characterized the Drosophila homolog of mammalian Jun-N-terminal kinases (DJNK). We show that DJNK is encoded by basket (bsk). Like hemipterous (hep), which encodes the Drosophila JNK kinase, $b s k$ is required in the embryo for dorsal closure, a process involving coordinate cell shape changes of ectodermal cells. Dorsal closure can also be blocked by dominant negative Drosophila cdc42, which has been shown to act upstream of JNKK in vertebrates. Therefore it appears that the JNK pathway is conserved and that it is involved in controlling cell morphogenesis in Drosophila. Although DJNK efficiently phosphorylates DJun in vitro, bsk function is not required for the specification of cell fate in the developing eye, a process that requires MAP kinase and DJun function.
\end{abstract}

[Key Words: Jun-N-terminal kinase; dorsal closure; basket; Drosophila; MAPK]

Received July 8, 1996; revised version accepted September 17, 1996.

Mitogen-activated protein kinase (MAPK) cascades transduce a variety of signals in eukaryotic cells in response to multiple extracellular stimuli. Depending on cell type, duration of stimulus, and pathway, they mediate a range of responses including proliferation, differentiation, and the regulation of metabolic pathways in differentiated cells (Marshall 1995). In yeast, for example, three different MAPK pathways have been identified, each one regulating different intracellular responses (Herskowitz 1995).

In vertebrate cells, much has been learned from biochemical studies of the extracellular-signal-regulated protein kinases (ERK) pathways and their involvement in growth control and cellular differentiation (for review, see Marshall 1994, 1995; Nishida and Gotoh 1993). In invertebrates, the corresponding MAPK pathway has been unraveled through the use of genetics in Drosophila (for review, see Dickson and Hafen 1993; Zipursky and Rubin 1994; Wassarman et al. 1995|, Caenorhabditis elegans (for review, see Kayne and Sternberg 1995; Kenyon 1995), and yeast (Herskowitz 1995). In Drosophila, this MAPK pathway (Rl/MAPK) is required for many processes during development, such as in the specification of terminal structures of the larva in response to the Torso receptor, in the formation of wing veins in response to the Drosophila EGF receptor homolog, and in

\footnotetext{
'Present address: Institute of Neuroscience, University of Oregon, Eugene, Oregon 97403 USA.

${ }^{2}$ Corresponding author.
}

the differentiation of photoreceptor cells in the compound eye (for review, see Domínguez and Hafen 1996).

By contrast, the role of the Jun- $\mathrm{N}$-terminal kinase (JNK) pathway, another MAPK pathway, is less well understood. In particular, there is a dearth of genetic studies of the JNK pathway in a multicellular organism, although recently a Drosophila JNK kinase has been reported (Glise et al. 1995). From studies in vertebrate cell culture systems the JNK pathway has been implicated in the response of cells to stress, growth factors, and Ras activation (Karin and Hunter 1995). In vitro, mammalian JNKs efficiently phosphorylate c-Jun on two serine residues (Ser63 and Ser73) in the amino-terminal domain of the protein. This phosphorylation correlates well with c-Jun activation. In contrast, ERKs seem to phosphorylate c-Jun at another site in the carboxy-terminal domain that correlates with inhibition of c-Jun DNA binding (Karin and Hunter 1995). Ras can activate both pathways, but to different potencies in several cell lines (Karin and Hunter 1995). Recent reports have suggested other interactions between the JNK and ERK pathways. For example, in rat PC- 12 cells, the JNK pathway plays a role in apoptosis, whereas the ERK pathway promotes cell survival (Xia et al. 1995). In contrast, in anergic T cells both pathways need to be activated to mediate the response of $\mathrm{T}$ cells to antigen presentation ( $\mathrm{Su}$ et al. 1994; Fields et al. 1996; Li et al. 1996). Because all these studies have been carried out in cultured cells, little is known about the role of the JNK pathway during the development of multicellular organisms and its interac- 
tion with other parallel MAP kinase pathways such as the ERK pathway in vivo.

To characterize the JNK pathway in a genetically amenable multicellular organism, we have cloned and characterized DJNK, the Drosophila homolog of mammalian JNKs. Here we show that DJNK is encoded by the basket (bsk) gene and that mutations in bsk, like mutations in the DJNKK gene hemipterous (hep) (Glise et al. 1995), disrupt dorsal closure, a process during embryonic development that involves cell shape changes and cytoskeletal reorganization (Young et al. 1993). We present evidence that the JNK and Rl/MAPK pathways in Drosophila are largely independent in the embryo and the eye. Our results also suggest that DJNK function is not required for Jun function in the eye.

\section{Results}

\section{Drosophila INK is a protein kinase}

From a staged embryonic cDNA library we isolated cDNAs whose deduced amino acid sequence exhibits high similarity to mammalian JNKs. Because of this, we named the encoded protein DINK (Drosophila Jun-Nterminal kinase). Northern blot analysis probed with the DINK cDNA revealed a single band of $1.5 \mathrm{~kb}$, the size of which corresponds to the longest cDNA (1507 bp) (data not shown).

The degree of amino acid identity between the Drosophila sequence and its mammalian counterparts is $>70 \%$ (Fig. 1A). In the kinase domain, DJNK contains all the conserved residues of protein kinases, and the TPY motif between kinase subdomains VII and VIII that is phosphorylated on the threonine and tyrosine residues to activate JNKs. The amino acid sequence between kinase subdomains IX and $\mathrm{X}$ varies between the JNKs, and determines the efficiency of binding to c-Jun (Kallunki et al. 1994). In this region, DJNK is most homologous to JNK2. In contrast, DJNK shares only $50 \%$ amino acid identity with the Drosophila Rolled (Rl) MAPK. This suggests that Rl/ERK MAPKs and JNKs diverged in evolution before the separation of vertebrates and invertebrates.

Next, we characterized DJNK biochemically. To do so, we performed in vitro kinase assays with Jun and myelin basic protein (MBP) as substrates. Western blot analysis of Drosophila extracts of embryos, larvae, pupae, and adults probed with anti-JNK1 antibody revealed a single band with an apparent molecular weight of $49 \mathrm{kD}$, the predicted weight of DJNK (Fig. 2A). This antibody specifically recognizes DJNK and does not cross-react with the similarly sized Rl/MAP kinase on Western blots (Fig. 2B). To test for DJNK protein kinase activity we immunoprecipitated DJNK and Rl/MAP kinase from larval protein extracts and performed in vitro kinase assays on the immunoprecipitates using DJun as a substrate (Fig. 2B). Like their vertebrate homologs, both DJNK and Rl/ MAP kinase phosphorylated DJun.

Finally, we wanted to test whether DJNK can be induced by stress, as it has been shown for mammalian
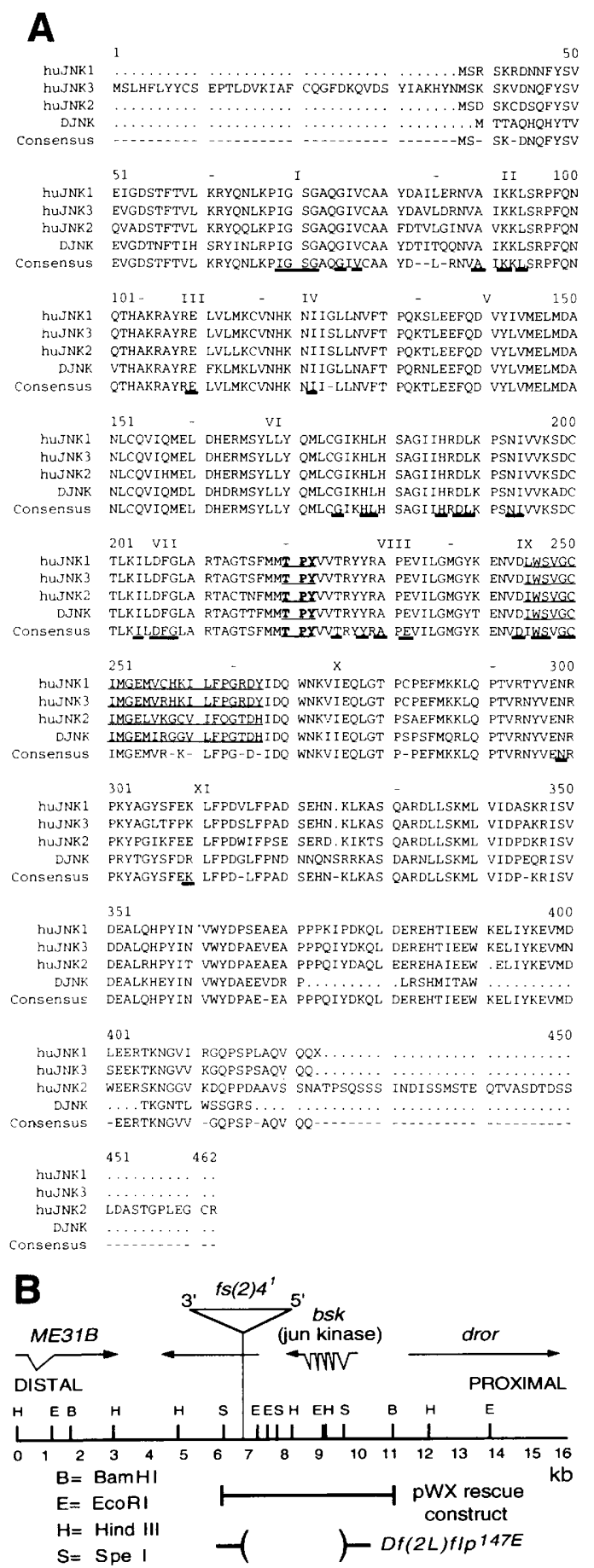

Figure 1. Molecular characterization of DJNK. $(A)$ Sequence alignment of DINK with mammalian JNKs. The conserved TPY motif is marked with bold underlines, the kinase subdomains are marked with roman numerals above the sequences, the conserved residues within the kinase domain are underlined in the consensus sequence, and the "specificity-determining region" domain is underlined in the four listed sequences. $(B)$ Molecular map of the $31 \mathrm{~B}$ region where the $D / N K$ is located. The $D I N K$ open reading frame encodes 354 amino acids and is split by five introns. It lies proximal to $f_{s}(2) 4$ and $M E 31 B$, and distal to the receptor tyrosine kinase gene dror. Below the genomic restriction map are diagrammed the extent of the pWX rescue construct and the extent of $D f(2 L) f l p 147 E$. 
A

\section{8}
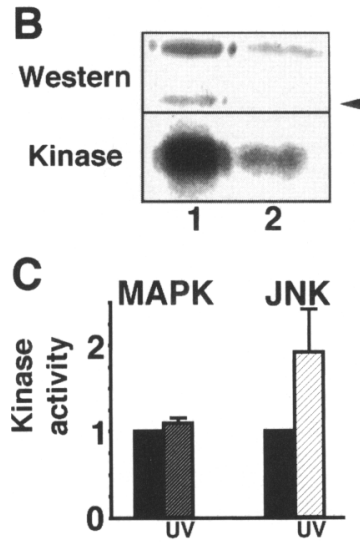
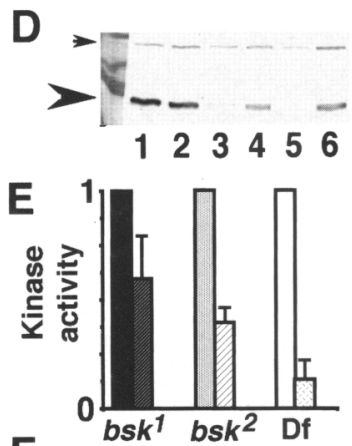

$\mathbf{F}$

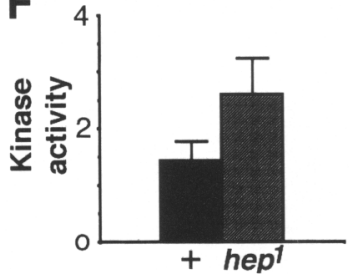

Figure 2. Biochemical characterization of DJNK. (A) Western blot of protein extracts of the following developmental stages probed with the JNK1 antibody; embryos (lane 1), first instar larvae (lane 2), second instar larvae (lane 3), early third instar larvae (lane 4), late third instar larvae (lane 5), pupae (lane 6), male flies (lane 7), and female flies (lane 8). For $A$ the molecular weight markers are $52 \mathrm{kD}, 35 \mathrm{kD}, 32 \mathrm{kD}$; for $D, 84 \mathrm{kD}, 62 \mathrm{kD}$, and $52 \mathrm{kD}$. (B) Kinase assay of larval lysates with DJun as a substrate. The lysate was split in two: One-half was precipitated with anti-JNKl antibody (lane 1), and the other half with antiRolled antibody (lane 2). The kinase assay blot was then probed with JNK1 antibody. The upper panel represents the anti-JNK1 antibody Western, showing that anti-JNKl antibody does not recognize Rolled, and the lower panel the corresponding kinase autoradiogram. The arrowhead marks the position of the DINK band. (C) Normalized kinase assays with either 5-min UVtreated larvae, or unirradiated controls. Irradiated and unirradiated lysates were split in two, and treated as in $B$. Normalization is to unirradiated controls, first column of each pair $(n=5)$. $(D)$ Western blot with JNK1 antibody of embryonic lysates from the following mutants: $b s k^{1} / b s k^{1}$ (lane 1$) ; b s k^{1} /+$ (lane 2); $b_{s k}{ }^{2} / b s k^{2}$ (lane 3); $b s k^{2} /+($ lane 4); Df(2L)flp147E/Df(2L)flp147E (lane 5); $D f(2 L) f l p 147 E /+$ (lane 6). The big arrowhead marks DJNK. As a control, the blot was also developed with anti-hsp83 antibodies (small arrowhead). (E) Normalized DJNK kinase activity in embryonic lysates of $b s k^{1}, b s k^{2}$, and $D f(2 L) f l p 147 E$ homozygotes normalized to their balancer siblings (first columns of each pair). $n=4$ for $b s k^{1}$ and $D f(2 L) f l p 147 E$, and $n=$ 3 for $b s k^{2}$. $(F)$ Normalized DJNK kinase activity in male/female wild-type larvae compared with male $h e p^{1}$ /sibling wild-type females larvae. $n=5$.

JNKs (Hibi et al. 1993; Dérijard et al. 1994; Engelberg et al. 1994; Kyriakis et al. 1994; Minden et al. 1994; Pombo et al. 1994). Third instar larvae were UV irradiated for 5 min, and after a period of recovery, used for kinase assays. Under these conditions DJNK activity was enhanced, whereas Rolled activity was not (Fig. 2C). These results show that DJNK is a protein kinase most akin to JNKs, and distinct from Rolled/MAPKs. Like its vertebrate homolog it phosphorylates DJun in vitro and is inducible by UV treatment.

\section{DINK is expressed dynamically during embryonic development}

To examine the pattern of DINK expression during embryogenesis we hybridized whole-mount embryos with an antisense RNA probe synthesized from a DJNK cDNA template. DINK is present homogeneously, albeit at low levels, in early syncitial stage embryos (Fig. 3A). Later, during gastrulation, high levels of DINK mRNA are detected in groups of cells that will undergo shape changes during morphogenetic movements: the cephalic furrow, the anterior and posterior transverse folds, and the leading edge of the ventrolateral epidermis (Fig. 3CF). Beginning at stage 8-9 of embryogenesis expression is also detected in the peripheral and central nervous system (CNS), and this expression is maintained in the CNS and imaginal disks during larval stages (Fig. 3B-F; data not shown). The dynamic and specific expression pattern of DJNK contrasts with the homogeneous expression pattern of other kinases, including Hep and Rl. In vertebrates, JNK3 expression is also specific: It is expressed in subsets of pyramidal cells in the CNS (Mohit et al. 1995).

\section{Mutations in basket disrupt DINK function}

The DINK gene is located in the $31 \mathrm{~B}$ region on the polytene chromosomes (data not shown). Molecular characterization of the region showed that $D I N K$ is flanked by two transcription units corresponding to the $f_{s}(2) 4$ and the $M E 31 B$ loci on the distal side, and the receptor tyrosine kinase gene dror (Wilson et al. 1993; A. Fritz unpubl.) on the proximal side (Fig. 1B). Among other mutations known to map to this region is basket (bsk) (Nüsslein-Volhard et al. 1984). Homozygous bsk mutant embryos die at the end of embryogenesis with a dorsal hole in their cuticle (Nüsslein-Volhard et al. 1984; Lindsley and Zimm 1992). Because mutations in hep, which encodes Drosophila JNKK, have a similar embryonic

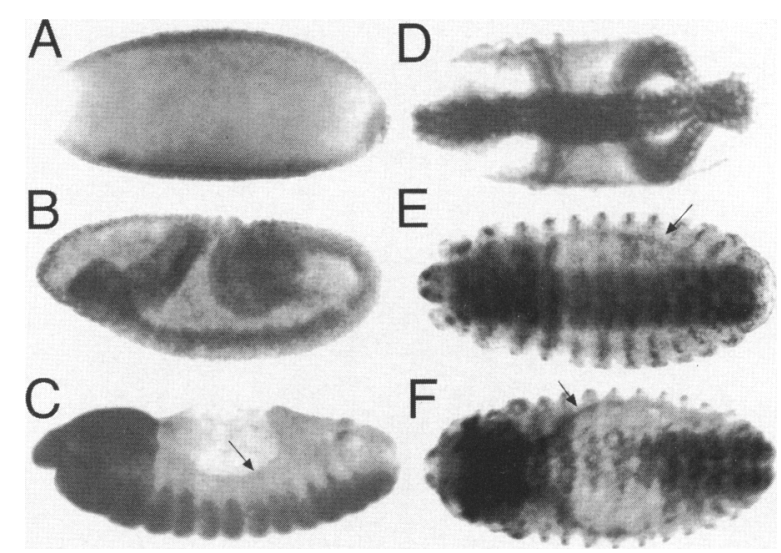

Figure 3. Localization of DINK mRNA in whole mount embryos. In all panels anterior is left, and in $A, B$, and $C$ dorsal is up. $D$ and $E$ are ventral views, and $F$ is a dorsal view. Arrows in $C, E$, and $F$ mark the leading edge of the ventrolateral epidermis. $(A)$ Stage 5 embryo; $(B, D)$ stage $7-8 ;(C, E, F)$ stage 12 . For nomenclature, see Campos-Ortega and Hartenstein (1985). 
phenotype, we wanted to test whether bsk encodes DJNK. To this end we performed transgenic rescue experiments in bsk mutant backgrounds with a $5-\mathrm{kb}$ genomic construct (pWX; Fig. 1B) that only includes the DJNK transcript. A single copy of the pWX construct was able to rescue to viable, fertile adults the lethality of heteroallelic combinations of $b s k$ mutants and deficiencies uncovering $b s k$. Because pWX does not include $d r o r$, $f_{s}(2) 4$, or $M E 31 B$ sequences, we conclude that the $b s k$ gene codes for DJNK.

The bsk alleles and deficiencies can be ordered in an allelic series with increasing strength of the cuticular phenotypes: $D f(2 L) / 27<b s k^{1}<b s k^{2}<D f(2 L) f 1 p 147 E$ (Fig. 4B-E). $D f(2 L) f 1 p 147 E$ is a small, 3-kb deletion generated by an imprecise excision event of the P-element insertion in $f_{s}(2) 4$ ( $\mathrm{K}$. Beckingham, pers. comm.). We found that $D f(2 L) f l p 147 E$ breaks proximal to the bsk gene, deleting most of the $b s k$ coding sequence including the kinase domain, and part of the $f_{S}(2) 4$ transcript. Therefore $D f(2 L) f l p 147 E$ is a null allele for $b s k$. In addition, we found that $b s k^{1}$ is temperature-sensitive. At $18^{\circ} \mathrm{C}$, egg lays show weaker phenotypes (Fig. 4F).

Next we examined the expression and activity of DJNK by Western blot analysis and in vitro kinase assays on extracts of $b s k$ mutants. $b s k^{1}$ mutant embryos still have wild-type levels of DJNK, whereas both $b s k^{2}$ and $D f(2 L) f l p 147 E$ show strong reductions in DJNK compared with their respective heterozygous siblings (Fig. 2D). Kinase activity from $b s k^{1}$ and $b s k^{2}$ mutant embryos

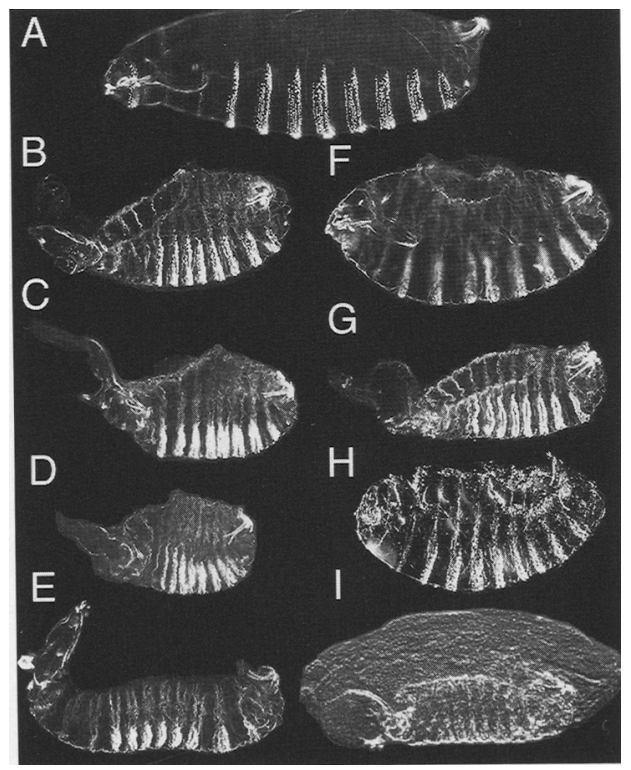

Figure 4. Allelic series of $b s k / D I N K$ mutant phenotypes. Cuticular preparation of embryos of the following genotypes: $(A)$ Wild-type; (B) $b s k^{1} / b s k^{1} ;$ (C) $b s k^{2} / b s k^{2} ;(D)$ Df(2L)flp147E/ $D f(2 L) f l p 147 E_{;}(E, I) b s k^{1} / D f(2 L) f l p 147 E$ derived from $b s k^{1} / b s k^{1}$ germ-line clones; $(F) b s k^{1} / b s k^{1}$ reared at $18^{\circ} \mathrm{C}_{;}|G| h_{e p}{ }^{1}$; and $\langle H\rangle$ UAS-Dcdc $42^{\mathrm{N} 17} /+$; B69-Gal $4 /+$. I was photographed before digestion with Hoyer's to show the embryonic tissues. In all panels anterior is left, and dorsal is up. (I) Ventrolateral view. was significantly reduced $160 \%$ and $40 \%$ of heterozygous siblings, respectively), whereas $D f(2 L) f I p 147 E$ mutant embryos had only $13 \%$ kinase activity of heterozygous siblings (Fig. 2E). The residual DJNK activity observed in the $D f(2 L) f l p 147 E$ homozygous embryos could be due to maternal contribution of Bsk product in the egg.

\section{basket shows maternal contribution and disrupts initiation of dorsal closure}

We examined the effect of removal of the maternal contribution by making germ-line clones using mitotic recombination. Embryos lacking both maternal and zygotic $b s k$ activity show an extreme dorsal open phenotype: Cuticle is formed only in the ventral and ventrolateral parts of the mutant embryo (Fig. 4E and I). This phenotype is stronger than phenotypes observed by removing only the zygotic bsk activity. We conclude that bsk has a maternal contribution. However, $b s k^{1} \mathrm{em}$ bryos derived from $b s k^{1}$ germ-line clones are rescued by zygotic $b s k^{+}$function to fertile adults. This indicates that the zygotic bsk expression can compensate for the lack of maternal product.

Dorsal closure occurs during mid-embryogenesis and involves cell shape changes but not cell division (Campos-Ortega and Hartenstein 1985). The cells of the leading edge of the ventrolateral epidermis elongate and stretch in the dorsoventral axis (followed by rows of epidermal cells underneath) until the two edges meet in the dorsal midline and close the embryo, effectively internalizing the cells of the amnioserosa. The epidermal cells stretch over the surface of the amnioserosa cells. Apical accumulation of actin and myosin in the leading edge cells initiates the final steps of dorsal closure (Young et al. 1993). The latter process is disrupted in strong zipper mutants. zipper codes for a nonmuscle myosin heavy chain (Young et al. 1993).

We stained bsk mutant embryos with antibodies against Spectrin and Coracle (Pesacreta et al. 1989; Fehon et al. 1994). Coracle is the Drosophila homolog of the vertebrate band 4.1 cytoskeletal protein (Fehon et al. 1994). Both anti Spectrin and Coracle antibodies mark the profiles of the cells. $b s k^{1}$ embryos show the initial features of dorsal closure: The leading edge cells change shape and elongate, but the process fails to come to completion, because the first rows underneath the leading edge cells either show only a partial change in shape, or fail to change cell shape completely (Fig. 5B). In wildtype embryos, the epidermal cells underneath the leading edge change shape and elongate after the leading edge cells do (Fig. 5A). Df(2L)flp147E embryos show a more extreme phenotype: The leading edge cells elongate even less than in $b s k^{1}$ mutants (Fig. 5C). Embryos derived from $b s k^{1}$ germ-line clones with no paternal contribution show no change in cell shape and dorsal closure never initiates (Fig. 5D). This result shows that bsk is required for the initiation of dorsal closure.

In bsk mutants, the spreading defect of the cells is more pronounced in anterior cells. In the posterior part of the embryo in weak mutants, some stretching epider- 

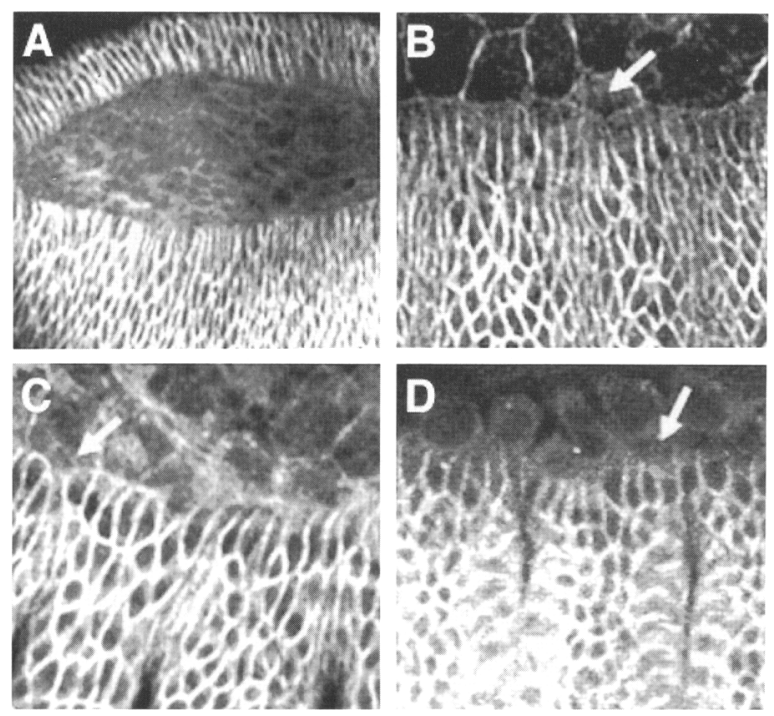

Figure 5. Cell shape changes during dorsal closure in bsk mutant embryos. (A) Wild-type larvae showing the ventrolateral epithelia during dorsal closure over the amnioserosa cells. $(B)$ $b s k^{1}$ embryo, where the leading edge cells (arrows in $B, C$, and $D$ ) and some underlying epithelial cells have begun elongation, but the process stopped midway because the epithelium is partially detached from the underlying amnioserosa cells (top, at left). $(C)$ $D f(2 L) f l p 147 E$ embryo showing only partial elongation of the leading edge cells. (D) $b s k^{1}$ germ-line clone where the leading edge cells have not undergone cell elongation (cf. $D$ with $B$ ). $A$ and $D$ were stained with anti coracle antibody, and $B, C$, and $D$ with anti spectrin antibody.

mal cells meet at the dorsal midline and effect closure, but not in the anterior part. As a consequence, the anterior portion remains open and the forming head structures and mouthparts are thrusted forward, together with parts of the gut and the CNS. Ventral furrow formation and other morphogenetic movements not directly affected by dorsal closure occur normally in the bsk mutants, showing that the defect is specific to dorsal closure. The graded series of bsk mutant phenotypes described here argues for a sustained requirement of bsk throughout the process of dorsal closure: Reductions in the amount of protein are reflected in a concomitant reduction in the extent of cell elongation. A similar conclusion was drawn from transgene rescue experiments of hep (Glise et al. 1995).

\section{Dominant negative Dcdc42 also shows a dorsal open phenotype}

The small GTPases Rac and Cdc42 have been implicated together with JNKK and JNK in the JNK pathway in vertebrates (Coso et al. 1995; Hill et al. 1995; Minden et al. 1995). In Drosophila, the homologs have been cloned (Dcdc42 and Drac), but no mutations have been isolated (Eaton et al. 1995). However, expression of a dominant negative form of DracA under the control of the heat shock promoter resulted in dorsal closure phenotypes
(Harden et al. 1995). We tested whether interfering with Dcdc42 function would also give a dorsal closure phenotype. We used a transgene that encodes a dominant negative form of Dcdc42 (UAS-Dcdc42 ${ }^{N 17}$ ) (Eaton et al. 1995) and expressed it under the control of Gal4 in the Gal4-69B line (Brand and Perrimon 1993). This line drives Gal4 expression in epidermal cells during embryogenesis (Brand and Perrimon 1993). Embryos with one copy of UAS-Dcdc42 $2^{N 17}$ and $69 B$ showed a dorsal open phenotype (Fig. $4 \mathrm{H}$ ). This result suggests that Dcdc42 like DracA acts in dorsal closure regulation. Therefore it is possible that Dedc42 and DracA function in the DINK pathway like their vertebrate homologs.

\section{bsk regulates puckered expression}

puckered (puc) mutations disrupt the formation of a suture between the two sides of the embryo in the dorsal midline. puc is expressed in the leading edge cells during dorsal closure, as revealed by lac $Z$ expression of an enhancer trap insertion in puc $\left(p u c^{E 69}\right)$ (Ring and MartinezArias 1993). In hep mutants, puc-lacZ is not expressed in the dorsal rim cells during dorsal closure, suggesting that expression of puc-lacZ is dependent on hep function (Glise et al. 1995). To test whether puc expression is also dependent on $b s k$ function we stained embryos from a cross of $D f(2 L) f l p 147 E /+$; puc-lacZ/ + and $b s k^{1} /+$ flies. From this cross half of the progeny carry the puclac $Z$ transgene and should show staining of the dorsal rim cells if bsk function were not required for puc expression. We found, however, that of 324 embryos at the stage of dorsal closure, only 92 showed detectable puc$1 a c Z$ staining. This number deviates significantly from the expected half that was observed in control crosses not involving $b s k$ alleles. In fact, these numbers fit a distribution of one-quarter staining and three-quarters nonstaining embryos $\left(\chi^{2}=1.99\right)$ and suggest that not only $b s k^{1} / D f(2 L) f l p 147 E$ embryos but also the embryos that are heterozygous for the $b s k$ null allele $[D f(2 L) f l p 147 E]$ lack detectable levels of lacZ staining. These results suggest that puc-lac $Z$ expression in the dorsal rim cells is dependent on hep and on $b s k$ function. It is interesting to note that it has been shown recently that puc encodes a member of the CL100 family of dual specificity MAP kinase phosphatases (A. Gampel, E. Martin-Blanco, and A. Martinez-Arias, pers. comm.). In vertebrates it has been shown that CL100 is transcriptionally induced by the activation of the MAP kinase pathway and stress (Keyse and Emslie 1992; Sun et al. 1993). Because puc expression is similarly regulated by the DINK pathway, it appears that yet another element of this pathway has been conserved.

We have also noted that in older bsk mutant embryos (scored by the $b s k$ cuticular phenotype) heterozygous for puc-lacZ, there is an ectopic expression of puc-lacZ, including cells of the amnioserosa (data not shown). This indicates that bsk function might be required later to represses puc-lac $Z$ expression. In this regard, it is interesting to note that DJNK activity is increased in extracts 
of hep ${ }^{1}$ mutant larvae (Fig. 2F). This result suggests that DJNK activity is not properly regulated in $h e p^{1}$ mutants.

bsk function is not required for cell fate specification in the eye

We have shown that DJNK phosphorylates DJun in vitro, Because DJun has been implicated in the specification of the R7 photoreceptor cells in the developing eye (Bohmann et al. 1994; Treier et al. 1995), we wanted to investigate a possible role of $b s k$ in photoreceptor cell specification. In situ hybridization experiments with a bsk RNA probe showed expression in the eye antennal disk (data not shown). We generated clones of homozygous $b s k^{1}$ cells in a heterozygous $b s k^{1}$ background in the eye by mitotic recombination. $b s k^{1}$ mutant cells proliferate normally because the clone size is similar to that of control clones. Furthermore, within the $b s k^{1}$ mutant clones of young flies ommatidia had developed normally. Only occasionally mutant ommatidia with altered number of photoreceptor cells are seen (Fig. 6). The specification of the R7 photoreceptor cell, which is most sensitive to the expression of a dominant negative DJun protein (Treier et al. 1995), is not affected in bsk mutant clones. Because $b s k^{1}$ is not a complete loss-of-function allele of $b s k$, it is possible that in the $b s k^{1}$ cells there is still sufficient JNK activity for normal Jun activation. We therefore tested also clones of $D f(2 L) f l p 147 E$. Cells homozygous for $D f(2 L) f l p 147 E$ lack not only bsk function but also $f s(2) 4$ function, which affects cell size (J. Riesgo-Escovar and E. Hafen, in prep.). Despite their reduced size, homozygous $D f(2 L) f l p 147 E$ cells were able to form normal ommatidial units with eight photoreceptor cells (data not shown). These results indicate that in absence of $b s k$ function specification of photoreceptor cells is normal.

\section{Discussion}

We have isolated and characterized a novel Jun kinase in

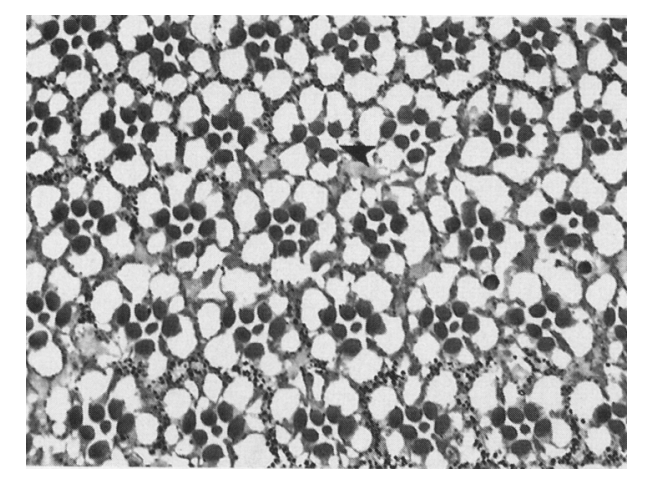

Figure 6. Analysis of bsk function in the developing eye. Tangential section, at the level of the R7 photoreceptor cell, through the eye of a $b s k^{1} /+$ fly carrying a clone of homozygous $b s k^{1}$ cells (marked by the absence of pigment granules). Note that most ommatidia in the clone are wild-type; typically only a few have abnormal numbers of photoreceptors (arrowhead).
Drosophila. Sequence analysis reveals high homology to its vertebrate counterparts, especially to JNK2. As is the case for its mammalian homologs, DJun is a good substrate for DJNK in vitro. We show that DINK is encoded by the bsk gene. The embryonic lethality associated with bsk loss-of-function alleles is fully rescued by a genomic rescue fragment encompassing only the DJNK transcription unit. DJNK protein levels and kinase activity are severely reduced in embryos homozygous for either the strong $b s k^{2}$ allele or the $D f(2 L) f l p 147 E$. Mutations in bsk and in hep (Glise et al. 1995) affect the process of dorsal closure, suggesting that both of these kinases are required together in this process.

\section{The role of Bsk in dorsal closure}

A large number of embryonic lethal mutations disrupt the process of dorsal closure during embryogenesis and display a common "dorsal open" phenotype (Jürgens et al. 1984; Nüsslein-Volhard et al. 1984; Wieschaus et al. 1984). Cloned genes in this group can be grouped into two main classes: genes involved in the regulation of dorsal closure, and genes coding for structural proteins required for dorsal closure.

The first class is represented by members of the JNK pathway: bsk and hep (Glise et al. 1995) and possibly DracA (Harden et al. 1995) and Dcdc42. Cleared cuticles of mutant embryos in this class show only dorsal closure phenotypes, whereas the body plan seems unaffected. Mutations in decapentaplegic (dpp), which encodes a TGF- $\beta$ homolog, or in the genes coding for its receptors, thick veins and punt, or schnurri, which encodes a transcription factor acting downstream of the Dpp receptors, also show dorsal open phenotypes (Jürgens et al. 1984; Nüsslein-Volhard et al. 1984; Affolter et al. 1994; Ruperte et al. 1995). It is unclear at present how the Dpp and the JNK pathway cooperate in this process.

The second class of mutants identifies genes coding for structural elements, mostly associated with the cytoskeleton, that are needed in dorsal closure. This group includes zipper, which encodes a nonmuscle myosin (Young et al. 1993); inflated, coding for an $\alpha$-integrin subunit (Wilcox et al. 1989); lethal(1)myospheroid, coding for a $\beta$-integrin subunit (Wieschaus et al. 1984); and coracle, which encodes a band 4.1 homolog (Fehon et al. 1994).

When is Bsk required during dorsal closure? We observe a wide range of dorsal open phenotypes depending on the strength of the mutant allele and the presence or absence of maternally derived Bsk. The strongest phenotype is observed when both maternal and zygotic Bsk are reduced. The resulting embryos fail to initiate the closing process. When only the zygotic component is removed the process initiates normally but is not completed. This argues strongly for a sustained requirement of bsk during dorsal closure, which normally lasts $\sim 2 \mathrm{hr}$.

Little is known about the exact role that hep and bsk play in the process of dorsal closure. Specifically, it is not clear what the target of JNK phosphorylation is in dorsal 
closure. JNK could directly phosphorylate and modify cytoskeletal components involved in dorsal closure such as zipper, coracle, inflated, and 1(1)myospheroid. Alternatively, JNK could modify the activity of transcription factors that are known to be involved in dorsal closure. Mutations in genes coding for several transcription factors have dorsal open phenotypes, like pannier (Ramain et al. 1993; Heitzler et al. 1996) and serpent (Abel et al. 1993; Frank and Rushlow 1996), two GATA transcription factors; and anterior open (Nüsslein-Volhard et al. 1984), an ETS domain protein (Rogge et al. 1995). The fact that both hep and bsk mutants affect the expression of puc as monitored by the puc-lacZ staining suggests that JNK acts by regulating transcription factors rather than by directly modifying cytoskeletal components involved in the actual process of cell shape change. Genetic and biochemical experiments are needed to identify the putative transcription factors whose activity is modulated by the JNK pathway as well as further target genes whose expression is modified by the pathway.

\section{Different developmental processes are controlled by the INK pathway and the MAP kinase pathway in Drosophila}

With the identification and cloning of $b s k$ and hep /Glise et al. 1995|, the JNK pathway is firmly established in Drosophila. Dominant negative versions of DracA (Harden et al. 1995) and Dcdc42 give also dorsal closure phenotypes. It is thus possible that they form part of the pathway, as in the vertebrate INK pathway. A more definitive placement of Drac and Dedc42 in the DINK pathway requires the availability of loss-of-function mutants of these genes and gain of function mutations in $h e p$ and $b s k$. In the absence of such mutants we have tested whether bsk mutants act as dominant suppressors of the gain of function phenotype obtained by expressing activated Dcdc42 (UAS-Dcdc ${ }^{12}$ ) in ectodermal cells in the embryo. Preliminary results suggest that removal of one copy of bsk suppresses partially the dominant phenotype of activated Dcdc42 (data not shown). These results are consistent with a model in which Dcdc42 acts via DJNK during embryogenesis.

The phenotypes of mutations in genes of the INK pathway are different from those of mutations in the Rl/MAP kinase pathway in both the embryo and the adult. In the embryo, bsk is involved in dorsal closure and $r l$ is required for signaling in the Torso pathway (Brunner et al. 1994b). In larvae, in contrast to $r l, b s k$ appears not to be required for cell proliferation and for cell fate specification in the eye. Therefore, it appears that during Drosophila development the INK and MAP kinase pathway are largely independent.

In vertebrate cell culture systems, the main substrate for JNK is the transcription factor Jun. We have shown that DJNK can also phosphorylate DJun efficiently in vitro. Whether DJun is also a major in vivo target of DJNK, however, is questionable. Mutations in DJun have not been identified yet, but through the use of dominant negative and constitutively active Jun proteins ex- pressed selectively in the developing eye it has been shown that DJun may function in the specification of neural fate in the $\mathrm{R} 7$ equivalence group in response to the Sevenless signaling pathway (Treier et al. 1995). If indeed bsk were the major kinase involved in the activation of DJun, we would expect impairment of photoreceptor cell specification in clones of homozygous $b s k$ cells. However, $b s k$ mutant cells differentiate largely into normal-looking ommatidia. Therefore it appears that DJNK is not involved in photoreceptor cell specification and is not required for Jun activation in the eye. This is consistent with recent biochemical evidence suggesting that DJun phosphorylation is dependent on the activity of the rolled/MAP kinase in Drosophila (Peverali et al. 1996).

\section{Materials and methods}

\section{Genetics}

$D f(2 L) f l p 147 E$ was a gift of $\mathrm{K}$. Beckingham (Rice University, Houston, TX|. $b s k^{1}$ and $b s k^{2}$ were obtained from the Tübingen stock center, $h e p^{1}, h e p^{r 75}$, and $p u c^{E 69}$ from S. Noselli /Centre National de Recherche Scientifique, Toulouse, France); the Gal4-69B line from K. Basler (University of Zürich, Switzerland), and $D f(2 L) / 2$ and $D f(2 L) / 27$ from the Indiana Stock Center. The UAS-Dcdc42N17 and UAS-Dcdc42 $2^{V 12}$ transgenic flies were from L. Luo (University of California, San Francisco).

Besides $D f(2 L) f l p 147 E, D f(2 L) J 2$ and $D f(2 L) / 27$, with approximately the same breakpoints (31B-32A), had been reported to uncover bsk (Nüsslein-Volhard et al. 1984; Clegg et al. 1993). We performed complementation tests between the deficiencies and the extant $b s k$ alleles in all pairwise combinations. Failure of complementation was observed in all cases except with $D f(2 L) / 27$. Viable transheterozygous with the bsk alleles and $D f(2 L) / 27$ were recovered. Egg lays derived from these crosses and from the balanced $D f(2 L) / 27$ stock, though, gave rise to a fraction of dead embryos with dorsal open phenotypes, indistinguishable from weak to intermediate $b s k$ phenotypes. We conclude that $D f(2 L) / 27$ shows partial complementation with bsk behaving as a weak $b s k$ allele. It is possible that $D f(2 L) / 27$ breaks distally near $b s k$, and disrupts regulatory sequences. The $b s k^{2}$ allele has a stronger dorsal open phenotype than $b s k^{1}$ but could not be used for the clonal analysis because it contains at least two additional closely linked lethal mutations on the same chromosome.

For rescue experiments we used three different insertions of pWX: one insertion on the second chromosome, and two on the third chromosome. The $p W X$ line with the insertion on the second was recombined onto the Df(2L)flp147E chromosome and then homozygosed. For the third chromosomal lines, flies heterozygous for a bsk allele or $D f(2 L) / 2 / C y O ; p W X /+$ were crossed to $D f(2 L) f l p 147 E / C y O$ and the progeny scored for viable transheterozygotes. We rescued the following mutant combinations: $D f(2 L) f l p 147 E / b s k^{1}$ and $D f(2 L) f l p 147 E / D f(2 L) / 2$ for one insertion, and $D f(2 L) f l p 147 E / b s k^{2}$ for the other.

From the crosses with $p W X$ insertions, rescued $D f(2 L) f l p 147 E /$ $D f(2 L) I 2 ; p W X /+$ and $p W X, D f(2 L) f l p 147 E$ also uncover $f_{s}(2) 4$ mutations and homozygous females were sterile, as expected. We also used a longer rescue construct $(10 \mathrm{~kb})$ in the $\mathrm{X}$ chromosome, $p W A X . p W A X$ contains the $f_{s}(2) 4$ transcript and $b s k$. $p W A X$ is sufficient to rescue both $b s k$ and $f s(2) 4$ alleles (J.R. Riesgo-Escovar and E. Hafen, in prep.).

A chi-square test was used to assess the distribution of stage 
8-15 embryos stained for lacZ expression from the cross $D f(2 L) f l p 147 E /+$; $p u c^{E 69} /+$ males to $b s k^{1} / C y O$ females.

\section{Clonal analysis}

Germ-line clones of $b s k^{1}$ were generated by use of the "dominant female sterile" technique (Hou et al. 1995) with the FLPFRT recombinase system (Xu and Rubin 1993). For $b s k^{2}$ and $D f(2 L) f l p 147 E$, the same procedure was followed, but eggs were not recovered. $D f(2 L) f l p 147 E$ also partially deletes a female sterile gene (J.R. Riesgo-Escovar and E. Hafen, in prep.). $b s k^{2}$ has additional mutations on the chromosome (J.R. Riesgo-Escovar and E. Hafen, unpubl.); this could account for the female sterility observed. We also generated $b s k^{1}$ and $D f(2 L) f l p 147 E$ mutant clones marked by the absence of the white gene marker using the FLP-FRT recombinase system of (Xu and Rubin 1993).

\section{Germ-line transformations}

A 10-kb genomic fragment (positions 1.2-11.5 in Fig. 1B) cloned in the pW8 transformation vector (Klemenz et al. 1987) was digested partially with SpeI and the appropriate fragment religated to generate pWX. Transgenic flies were generated according to Basler et al. (1991).

\section{Cuticle preparations}

Embryos were dechorionated in $50 \%$ chlorox, devitellinized, washed, and mounted in Hoyer's medium. Cuticle preparations were photographed on a Zeiss Axiophot under bright and dark fields.

\section{Histology}

For antibody stainings, embryos were treated as described (Young et al. 1991). Anti Spectrin antibodies were used at a $1: 500$ dilution, anti-Coracle at 1:250. Fluorescently labeled secondary antibodies (Jackson, Inc.) were used at a 1:100 dilution. Coracle protein distribution in bsk mutant embryos is normal when both maternal and zygotic bsk function is removed. Mounted embryos were viewed on a Multiprobe laser confocal microscope (Molecular Dynamics, Inc.). Eye sections were done according to Brunner et al. (1994b). Images were assembled using Adobe Photoshop 3.0 software.

In situ hybridizations were performed essentially as described (Tautz and Pfeifle 1989) for embryos and larval brains using an antisense RNA probe encompassing the entire DINK cDNA. Sense probes were used in parallel as controls.

\section{Molecular techniques}

Standard molecular techniques were used (Sambrook et al. 1989). cDNAs and the corresponding genomic region were isolated, mapped, and sequenced on both strands using Sequenase according to the manufacturer's instructions with the use of several sequencing primers. Data was assembled and analyzed using the GCG software (Wisconsin).

\section{Kinase assays}

Anti-JNKl antibody was from Santa Cruz Biotechnology, Inc. MBP immune complex kinase assays were done essentially as described (Brunner et al. 1994a), except that for DJNK assays, 5 $\mu \mathrm{l}$ of JNK1 antibody was used per sample, and protein A-Sepharose beads, antibody, and lysates were added at the same time. Larval, pupal or embryonic extracts were homogenized in a ho- mogenizer adapted with a plastic Eppendorf tube pestle by six steps in RIPA buffer [phosphate buffered saline (PBS), 1\% Nonidet P40, 0.5\% sodium deoxycholate, and $0.1 \%$ SDS, to which was added every time fresh $10 \mathrm{mg} / \mathrm{ml}$ phenyl methyl sulfonyl fluoride (PMSF) in propanol $(10 \mu \mathrm{l})$, aprotinin $(30 \mu \mathrm{l} / \mathrm{ml})$ and 100 mM sodium orthovanadate]. For Westerns, we used a 1:1000 dilution of the JNK1 antibody.

DINK immunoprecipitated activity is concentration-dependent, and saturates between 20 and $30 \mathrm{~min}$ of incubation /data not shown). For UV treatment experiments, larvae were collected in empty glass vials with moist filter discs, and subjected to UV light from a transilluminator for $5 \mathrm{~min}$ at a $1-\mathrm{cm}$ distance. The vials were then removed, and larvae allowed to recover $(45$ min) before processing. Harsher UV treatments ( $15 \mathrm{~min}$ ) inhibit DINK by $50 \%$, and enhance Rolled activity $(100 \%) / n=3$ assays) (data not shown). For kinase assays in a hep ${ }^{1}$ background, we took advantage of the fact that $h e p^{1}$ hypomorphs are semilethal. We made a stock of $h e p^{1}$ and an attached X chromosome balancer, such that males are always $h e p^{1}$, and females are always wild-type. We separated male and female third instar larvae from the same cultures for kinase assays.

\section{Acknowledgments}

We thank K. Beckingham for providing the $D f(2 L) f l p 147 E$ stock and for sharing unpublished results. We thank C. Wilson, S. Noselli, K. Mathews, and the Tübingen Stock center for fly stocks. We thank R. Fehon for the Coracle antibody; K. Basler, B. Dickson, M. Domínguez, K. Dücker, S. Leevers, and I. Rodriguez for critical reading of the manuscript; and members of the Hafen lab (including S. Leevers and L. MacDougall) for discussions and constructive criticisms. This work was supported by grants from the Human Frontiers Science Organization to J.R.R. and a grant from the Swiss National Science Foundation to E.H.

The publication costs of this article were defrayed in part by payment of page charges. This article must therefore be hereby marked "advertisement" in accordance with 18 USC section 1734 solely to indicate this fact.

\section{Note}

The DINK cDNA sequence has been submitted to GenBank under accession no. U73196.

\section{References}

Abel, T., A.M. Michelson, and T. Maniatis. 1993. A Drosophila GATA family member that binds to Adh regulatory sequences is expressed in the developing fat body. Development 119: 623-633.

Affolter, M., D. Nellen, U. Nussbaumer, and K. Basler. 1994. Multiple requirements for the receptor serine/threonine $\mathrm{ki}$ nase thick veins reveal novel functions of TGF $\beta$ homologs during Drosophila embryogenesis. Development 120: 31053117.

Basler, K., B. Christen, and E. Hafen. 1991. Ligand-independent activation of the sevenless receptor tyrosine kinase changes the fate of cells in the developing Drosophila eye. Cell 64: 1069-1082.

Bohmann, D., M.C. Ellis, L.M. Staszewski, and M. Mlodzik. 1994. Drosophila Jun mediates Ras-dependent photoreceptor determination. Cell 78: 973-986.

Brand, A.H. and N. Perrimon. 1993. Targeted gene expression as a means of altering cell fates and generating dominant phenotypes. Development 118: 401-415. 
Brunner, D., K. Dücker, N. Oellers, E. Hafen, H. Scholz, and C. Klämbt. 1994a. The ETS domain protein Pointed-P2 is a target of MAP kinase in the Sevenless signal transduction pathway. Nature 370: 386-389.

Brunner, D., N. Oellers, J. Szabad, W.H. Biggs III, S.L. Zipursky, and E. Hafen. 1994b. A gain of function mutation in Drosophila MAP kinase activates multiple receptor tyrosine kinase signalling pathways. Cell 76: $875-888$

Campos-Ortega, J. and V. Hartenstein. 1985. The embryonic development of Drosophila melanogaster. Springer-Verlag, Berlin, Germany.

Clegg, N.J., I.P. Whitehead, J.K. Brock, D.A. Sinclair, R. Mottus, G. Stromotich, M.J. Harrington, and T.A. Grigliatti. 1993. A cytogenetic analysis of chromosomal region 31 of Drosophila melanogaster. Genetics 134: 221-230.

Coso, O.A., M. Chiariello, J.-C. Yu, H. Teramoto, P. Crespo, N. $\mathrm{Xu}, \mathrm{T}$. Miki, and J.S. Gutkind. 1995. The small GTP-binding proteins Racl and Cdc42 regulate the activity of the JNK/ SAPK signalling pathway. Cell 81: 1137-1146.

Dérijard, B., M. Hibi, I.H. Wu, T. Barrett, B. Su, T. Deng, M. Karin, and R.J. Davis. 1994. JNK1: A protein kinase stimulated by UV light and $\mathrm{Ha}$-Ras that binds and phosphorylates the c-Jun activation domain. Cell 76: 1025-1037.

Dickson, B. and E. Hafen. 1993. Genetic dissection of eye development in Drosophila. In The development of Drosophila melanogaster (ed. A. Martinez-Arias and M. Bate), pp. 13271362. Cold Spring Harbor Laboratory Press, Cold Spring Harbor, NY.

Domínguez, M. and E. Hafen. 1996. Genetic dissection of cell fate specification in the developing eye of Drosophila. Semin. Cell Dev. Biol. 7: 219-226.

Eaton, S., P. Auvinen, L.Q. Luo, Y.N. Jan, and K. Simons. 1995. CDC42 and Racl control different actin-dependent processes in the Drosophila wing disc epithelium. J. Cell Biol. 131: 151-164.

Engelberg, D., C. Klein, H. Martinetto, K. Struhl, and M. Karin. 1994. The UV response involving the Ras signaling pathway and AP-1 transcription factors is conserved between yeast and mammals. Cell 77: 381-390.

Fehon, R.G., I.A. Dawson, and T.S. Artavanis. 1994. A Drosophila homologue of membrane-skeleton protein 4.1 is associated with septate junctions and is encoded by the coracle gene. Development 120: 545-557.

Fields, P.E., T.F. Gajewski, and F.W. Fitch. 1996. Blocked Ras activation in anergic CD4 $+T$ cells. Science 271: 12761278.

Frank, L.H. and C. Rushlow. 1996. A group of genes required for the maintenance of the amnioserosa tissue in Drosophila. Development 122: 1343-1352.

Glise, B., H. Bourbon, and S. Noselli. 1995. hemipterous encodes a novel Drosophila MAP kinase kinase, required for epithelial cell sheet movement. Cell 83: 451-462.

Harden, N., H.Y. Loh, W. Chia, and L. Lim. 1995. A dominant inhibitory version of the small GTP-binding protein Rac disrupts cytoskeletal structures and inhibits developmental cell shape changes in Drosophila. Development 121: 903 914.

Heitzler, P., M. Haenlin, P. Ramain, M. Calleja, and P. Simpson. 1996. A genetic analysis of pannier, a gene necessary for viability of dorsal tissues and bristle positioning in Drosophila. Genetics 143: 1271-1286.

Herskowitz, I. 1995. MAP kinase pathways in yeast: For mating and more. Cell 80: 187-197.

Hibi, M., A. Lin, T. Smeal, A. Minden, and M. Karin. 1993. Identification of an oncoprotein- and UV-responsive protein kinase that binds and potentiates the c-Jun activation do- main. Genes \& Dev. 7: 2135-2148.

Hill, C.S., J. Wynne, and R. Treisman. 1995. The Rho family GTPases RhoA, Racl, and Cdc $42 \mathrm{Hs}$ regulate transcriptional activation by SRF. Cell 81: 1159-1170.

Hou, X.S., T.-B. Chou, M.B. Melnick, and N. Perrimon. 1995. The torso receptor tyrosine kinase can activate Raf in a Rasindependent pathway. Cell 81: 63-71.

Jürgens, G., C. Nüsslein-Volhard, and H. Kluding. 1984. Mutations affecting the pattern of the larval cuticle in Drosophila melanogaster II. Zygotic loci on the third chromosome. Roux's Arch. Dev. Biol. 193: 183-295.

Kallunki, T., B. Su, I. Tsigelny, H.K. Sluss, B. Dérijard, G. Moore, R. Davis, and M. Karin. 1994. JNK2 contains a specificity-determining region responsible for efficient c-Jun binding and phosphorylation. Genes \& Dev. 8: 2996-3007.

Karin, M. and T. Hunter. 1995. Transcriptional control by protein phosphorylation: Signal transmission from the cell surface to the nucleus. Curr. Biol. 5: 747-757.

Kayne, P.S. and P.W. Sternberg. 1995. Ras pathways in Caenorhabditis elegans. Curr. Opin. Genet. Dev. 5: 38-43.

Kenyon, C. 1995. A perfect vulva every time: Gradients and signaling cascades in C. elegans. Cell 82: 171-174.

Keyse, S.M. and E.A. Emslie. 1992. Oxidative stress and heat shock induce a human gene encoding a protein-tyrosine phosphatase. Nature 359: 644-647.

Klemenz, R., U. Weber, and W.J. Gehring. 1987. The white gene as a marker in a new P-element vector for gene transfer in Drosophila. Nucleic Acids Res. 15: 3947-3959.

Kyriakis, J.M., P. Banerjee, E. Nikolakaki, T. Dai, E.A. Rubie, M.F. Ahmad, J. Avruch, and J.R. Woodgett. 1994. The stressactivated protein kinase subfamily of c-Jun kinases. Nature 369: 156-160.

Li, W., C.D. Whaley, A. Mondino, and D.L. Mueller. 1996. Blocked signal transduction to the ERK and JNK protein kinases in anergic CD4 + T cells. Science 271: 1272-1276.

Lindsley, D.L. and G.G. Zimm. 1992. The genome of Drosophila melanogaster. Academic Press, San Diego, CA.

Marshall, C.J. 1994. MAP kinase kinase kinase, MAP kinase kinase and MAP kinase. Curr. Opin. Genet. Dev. 4: 82-89. . 1995. Specificity of receptor tyrosine kinase signaling Transient versus sustained extracellular signal-regulated $\mathrm{ki}$ nase activation. Cell 80: 179-185.

Minden, A., A. Lin, T. Smeal, B. Dérijard, M. Cobb, R. Davis, and M. Karin. 1994. c-Jun N-terminal phosphorylation correlates with activation of the JNK subgroup but not the ERK subgroup of mitogen-activated protein kinases. Mol. Cell. Biol. 14: 6683-6688.

Minden, A., A. Lin, F.-X. Claret, A. Abo, and M. Karin. 1995. Selective activation of the JNK signalling cascade and c-Jun transcriptional activity by the small GTPases Rac and Cdc42Hs. Cell 81: 1147-1157.

Mohit, A.A., J.H. Martin, and C.A. Miller. 1995. p49 3F12 kinase: A novel MAP kinase expressed in a subset of neurons in the human nervous system. Neuron 14: 67-78.

Nishida, E. and Y. Gotoh. 1993. The MAP kinase cascade is essential for diverse signal transduction pathways. Trends Biochem. Sci. 18: 128-131.

Nüsslein-Volhard, C., E. Wieschaus, and H. Kluding. 1984. Mutations affecting the pattern of the larval cuticle in Drosophila melanogaster. I. Zygotic loci on the second chromosome. Roux's Arch. Dev. Biol. 193: 267-282.

Pesacreta, T.C., T.J. Byers, R. Dubreuil, D.P. Kiehart, and D. Branton. 1989. Drosophila spectrin: The membrane skeleton during embryogenesis. J. Cell Biol. 108: 1697-1710.

Peverali, F.A., A. Isaksson, A.G. Papavassiliou, P. Plastina, L.M. Staszewski, M. Mlodzik, and D. Bohmann. 1996. Phospho- 
rylation of Drosophila jun by the MAP kinase rolled regulates photoreceptor differentiation. EMBO J. 15: 3943-3950.

Pombo, C.M., J.V. Bonventre, J. Avruch, J.R. Woodgett, J.M. Kyriakis, and T. Force. 1994. The stress-activated protein kinases are major c-Jun amino-terminal kinases activated by ischemia and reperfusion. J. Biol. Chem. 269: 26546-26551.

Ramain, P., P. Heitzler, M. Haenlin, and P. Simpson. 1993. pannier, a negative regulator of achaete and scute in Drosophila, encodes a zinc finger protein with homology to the vertebrate transcription factor GATA-1. Development 119: 1277 1291.

Ring, J.M. and A. Martinez-Arias. 1993. puckered, a gene involved in position-specific cell differentiation in the dorsal epidermis of the Drosophila larva. Development 119: 251259.

Rogge, R., P.J. Green, J. Urano, S. Horn-Saban, M. Mlodzik, B.-Z. Shilo, V. Hartenstein, and U. Banerjee. 1995. The role of yan in mediating the choice between cell division and differentiation. Development 121: 3947-3958.

Ruperte, E., T. Marty, D. Nellen, M. Affolter, and K. Basler. 1995. An absolute requirement for both type II and type I receptors, Punt and Thick Veins, for Dpp signalling in vivo. Cell 80: 889-897.

Sambrook, J., E.F. Fritsch, and T. Maniatis. 1989. Molecular cloning: A laboratory manual, 2nd edition. Cold Spring Harbor Laboratory Press, Cold Spring Harbor, NY.

Su, B., E. Jacinto, M. Hibi, T. Kallunki, M. Karin, and N.Y. Ben. 1994. JNK is involved in signal integration during costimulation of T lymphocytes. Cell 77: 727-736.

Sun, H., C.H. Charles, L.F. Lau, and N.K. Tonks. 1993. MKP-1 (3CH134), an immediate early gene product, is a dual specificity phosphatase that dephosphorylates MAP kinase in vivo. Cell 75: 487-493.

Tautz, D. and C. Pfeifle. 1989. A non-radioactive in situ hybridization method for the localization of specific RNAs in Drosophila embryos reveals translational control of the segmentation gene hunchback. Chromosoma 98: 81-85.

Treier, M., D. Bohmann, and M. Mlodzik. 1995. Jun cooperates with the ETS-domain protein pointed to induce photoreceptor R7 fate in the Drosophila eye. Cell 83: 753-760.

Wassarman, D.A., M. Therrien, and G.M. Rubin. 1995. The Ras signaling pathway in Drosophila. Curr. Opin. Genet. Dev. 5: 44-50.

Wieschaus, E., C. Nüsslein-Volhard, and G. Jürgens. 1984. Mutations affecting the pattern of the larval cuticle in Drosophila melanogaster III. Zygotic loci on the X-chromosome and the fourth chromosome. Roux's Arch. Dev. Biol. 193: 296-307.

Wilcox, M., A. DiAntonio, and M. Leptin. 1989. The function of PS integrins in Drosophila wing morphogenesis. Development 107: 891-897.

Wilson, C., D.C.I. Goberdhan, and H. Steller. 1993. Dror, a potential neurotrophic receptor gene, encodes a Drosophila homolog of the vertebrate Ror family of Trk-related receptor tyrosine kinases. Proc. Natl. Acad. Sci. 90: 7109-7113.

Xia, Z., M. Dickens, J. Raingeaud, R.G. Davis, and M.E. Greenberg. 1995. Opposing effects of ERK and JNK-P38 MAP kinases on apoptosis. Science 270: 1326-1331.

$\mathrm{Xu}, \mathrm{T}$. and G. Rubin. 1993. Analysis of genetic mosaics in developing and adult Drosophila tissues. Development 117: 1223-1237.

Young, P.E., T.C. Pesacreta, and D.P. Kiehart. 1991. Dynamic changes in the distribution of cytoplasmic myosin during Drosophila embryogenesis. Development 111: 1-14.

Young, P.E., A.M. Richman, A.S. Ketchum, and D.P. Kiehart. 1993. Morphogenesis in Drosophila requires nonmuscle my- osin heavy chain function. Genes \& Dev. 7: 29-41.

Zipursky, L.S. and G.M. Rubin. 1994. Determination of neuronal cell fate: Lessons from the R7 neuron in Drosophila. Annu. Rev. Neurosci. 17: 373-397. 


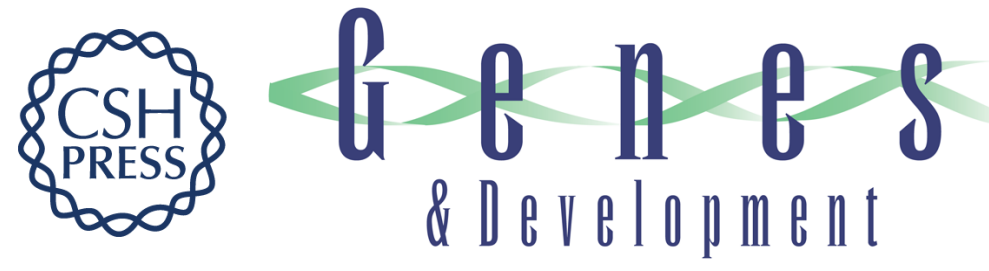

\section{The Drosophila Jun-N-terminal kinase is required for cell morphogenesis but not for DJun-dependent cell fate specification in the eye.}

J R Riesgo-Escovar, M Jenni, A Fritz, et al.

Genes Dev. 1996, 10:

Access the most recent version at doi:10.1101/gad.10.21.2759

References This article cites 59 articles, 25 of which can be accessed free at:

http://genesdev.cshlp.org/content/10/21/2759.full.html\#ref-list-1

License

Email Alerting Service

Receive free email alerts when new articles cite this article - sign up in the box at the top right corner of the article or click here.

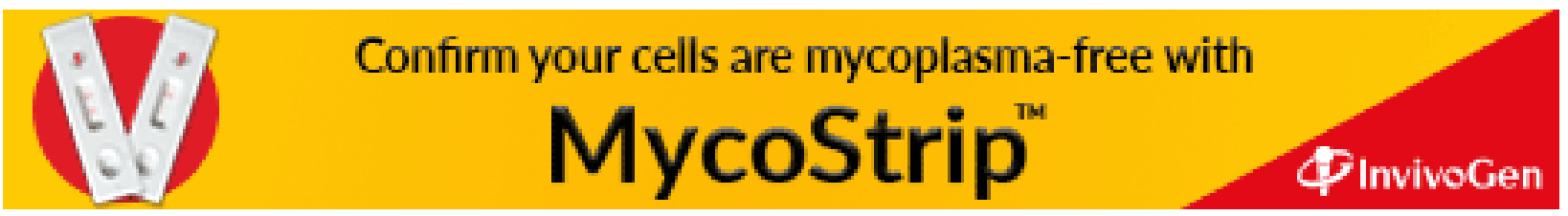

